\section{MYOCARDIAL FUNCTION IN OFFSPRINGS 5-8 YEARS AFTER PREGNANCY COMPLICATED BY PREECLAMPSIA OR DIABETES MELLITUS}

\author{
D. Fugelseth ${ }^{1}$, H. Ramstad ${ }^{1}$, A.S. Kvehaugen², \\ E. Nestaas ${ }^{3}$, A. Støylen ${ }^{4}$, A.C. Staff ${ }^{2}$
}

${ }^{1}$ Dept. of Neonatal Intensive Care Medicine, Oslo University Hospital, ${ }^{2}$ Dept. of Obstetrics and Dept. of Gynaecology, Oslo University Hospital, Ullevål, Oslo, ${ }^{3}$ Dept. of Paediatrics, Vestfold Hospital Trust, Tønsberg, ${ }^{4}$ Dept. of Cardiology and Dept. of Circulation and Medical Imaging, St. Olavs Hospital and Faculty of Medicine, Norwegian

University of Science and Technology, Trondheim, Norway

Background and aims: Preeclampsia and diabetic pregnancies share many pathophysiological features suggested to influence epigenetic changes during foetal life with importance for childhood development and later metabolic and cardiovascular diseases. Our objective was to study early myocardial affections in offsprings after these pregnancy complications.

Design: Forty-five children delivered (2001-2004) due to pregnancies complicated by preeclampsia $(n=25)$, diabetic mellitus type $1(n=8)$ and gestational diabetes mellitus $(n=12)$ were included at age 5-8 year (2008-2009). Fifteen children from uneventful pregnancies served as controls. Global systolic and diastolic longitudinal myocardial functions of right and left ventricle were examined by conventional Doppler and Tissue Doppler echocardiographic imaging (TDI) including assessment of annular velocities, displacement and myocardial wall deformation by strain and strain rate analyses. Offline analyses were performed by the Echopac PC software system.

Results: No major differences were found in myocardial function between the offsprings of pregnancy complications and control group. By pulsed wave and colour TDI, an increased late diastolic velocity ( $A^{\prime}$-wave) was found at mitral valve attachments in the preeclampsia vs. control and diabetic groups $(p \leq 0.01)$.

Conclusion: No major differences in myocardial function in offsprings after pregnancies complicated by preeclampsia or diabetes mellitus compared with controls were detected at 5-8 years of age. The numbers of examined children in each group were limited, and the study was not powered to detect minor differences in myocardial function. The increased late diastolic velocity in the preeclampsia group should be prospectively studied to assess whether it represents an early sign of diastolic dysfunction.

\section{8}

\section{MAJOR NEONATAL CONGENITAL HEART DISEASE IN IRELAND IS INCREASED IN INCIDENCE AND COMPLEXITY COMPARED TO INTERNATIONAL FIGURES}

\author{
S. Smith ${ }^{1}$, L. $\mathrm{Ng}^{1}, \mathrm{~A}$. Getty ${ }^{1}, \mathrm{M}$. Lavelle ${ }^{1}$, \\ E. Molloy², O. Franklin ${ }^{1}$
}

${ }^{1}$ Cardiology, Our Lady's Hospital for Sick Children, ${ }^{2}$ Neonatology, National Maternity Hospital Holles Street, Dublin, Ireland

Background and aims: The importance of ultrasound screening for major congenital heart disease(MCHD) is well established. In the Republic of Ireland termination of pregnancy for fetal abnormality is legally prohibited. Screening for congenital cardiac abnormality is offered in tertiary obstetric units but is not universally available. The study aimed to assess incidence of MCHD particularly of four chamber lesions in this context.

Methods: In 2009115 term infants were admitted to the National Children's Heart Centre in Our Lady's Children's Hospital in Dublin with MCHD defined as duct dependent lesions or lesions requiring surgery in the first 6 weeks of life. Their lesions were coded using the paediatric European Coding system and the diagnoses compared with EUROCAT (European Surveillance of Congenital Anomalies) data. Preterm infants and infants who were admitted outside the 6 week window were excluded.

Results: There were 75,720 live births in Ireland in 2009, of these 115 infants had MCHD. Of these $44(21 \%)$ had their diagnoses made antenatally. No patient with an antenatal diagnosis had a termination of pregnancy. The incidence of congenital heart disease in Irish newborns was significantly elevated for hypoplastic left and right heart, coarctation and pulmonary atresia.

Conclusion: Irish rates of antenatal diagnosis compare poorly with Europe. The severity and complexity of cardiac disease in Irish neonates is increased resulting in an increased demand on neonatal cardiac and cardiothoracic surgery services. 